\title{
Identifying links between origami and compliant mechanisms
}

\author{
H. C. Greenberg, M. L. Gong, S. P. Magleby, and L. L. Howell \\ Department of Mechanical Engineering, Brigham Young University, Provo, UT 84602, USA
}

Received: 25 February 2011 - Revised: 26 July 2011 - Accepted: 20 November 2011 - Published: 12 December 2011

\begin{abstract}
Origami is the art of folding paper. In the context of engineering, orimimetics is the application of folding to solve problems. Kinetic origami behavior can be modeled with the pseudo-rigid-body model since the origami are compliant mechanisms. These compliant mechanisms, when having a flat initial state and motion emerging out of the fabrication plane, are classified as lamina emergent mechanisms (LEMs). To demonstrate the feasibility of identifying links between origami and compliant mechanism analysis and design methods, four flat folding paper mechanisms are presented with their corresponding kinematic and graph models. Principles from graph theory are used to abstract the mechanisms to show them as coupled, or inter-connected, mechanisms. It is anticipated that this work lays a foundation for exploring methods for LEM synthesis based on the analogy between flat-folding origami models and linkage assembly.
\end{abstract}

Keywords. Compliant mechanisms, Lamina emergent mechanisms, Origami, Orimimetics

\section{Introduction}

Origami is the art of folding paper where ori- means fold and -kami means paper. The scope and complexity of origami has exploded in the last twenty years creating many different schools of thought (Demaine et al., 2010). Recently in the fields of science, mathematics and engineering, origami has been used to solve complex problems such as airbag folding, shock absorption (crash box), and deployable telescopic lenses (Cromvik, 2007; Ma and You, 2010; Heller, 2003). Origami design is governed by mathematical laws, which if better understood, could be applied in engineering. Specifically, the authors propose that origami can provide inspiration for synthesis techniques for compliant mechanisms. This paper identifies key concepts that can link origami to mechanism design.

Traditionally origami has been static and representational. However, kinetic origami focuses on origami models that have some novel motion. While the term "action origami" is a more generally known term, we introduce the term kinetic origami to refer to models that exhibit a mechanical motion and to differentiate from models such as paper airplanes which are also referred to as action origami. Figure 1 shows two examples, where one is a static origami structure

Correspondence to: S. P. Magleby (magleby@byu.edu) and the other is an action origami mechanism called a flasher hat; it is shown in its fabricated and deployed forms. The way an origami model folds also provides insight and another way to classifying origami. The class of rigidly foldable origami focuses on motion where the creases often act as joints and the faces act as links with bending stress only occurring at the creases (Tachi, 2006; Balkcom and Mason, 2008; Hull, 1994; Watanabe and Kawaguchi, 2006).

The process of folding origami has been examined using kinematic theory (Dai and Jones, 2002; Balkcom and Mason, 2008; Tachi, 2006; Buchner, 2003). This paper focuses on the motion of a finished origami and not the states in between flat and final states.

Since origami relies on the deflection of flexible materials it is a compliant mechanism. The origami mechanisms examined herein are all flat-folding in their final-folded state, and so they are part of a subgroup of compliant mechanisms called lamina emergent mechanisms (LEMs) (Jacobsen et al., 2010). Lamina emergent mechanisms are compliant mechanisms made from planar materials (lamina) with motion that emerges out of the fabrication plane.

A productive connection between origami and compliant mechanisms can be developed by drawing upon principles from graph theory to depict the origami mechanisms via simple, planar connected graphs. These graphs can show how the folds and facets interact with each other in their motion. Graphs allow for improved understanding of the interaction 


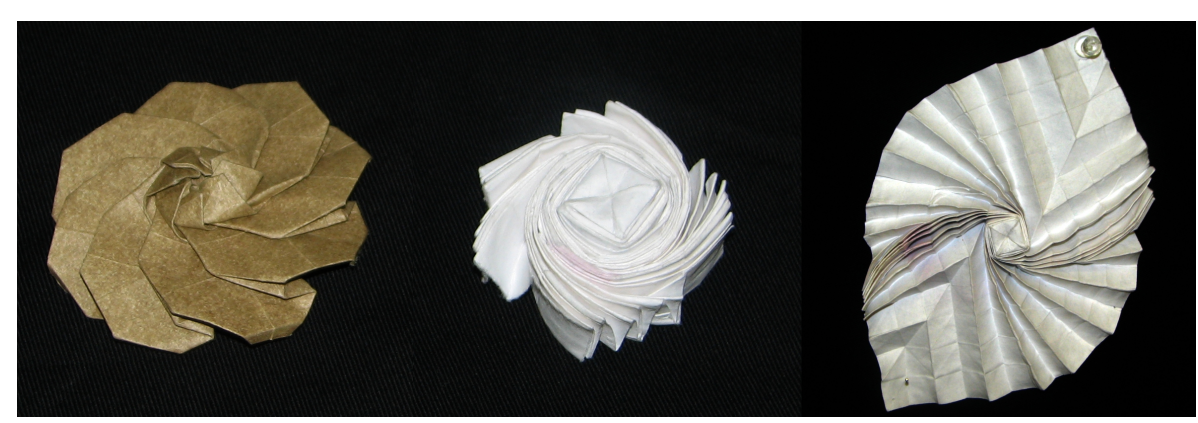

Figure 1. Example of an origami structure (left) and flasher hat mechanism in its fabricated (middle) and deployed form (right).

between motion and structure of origami, and can help in understanding how to predict complex motion and develop corresponding mechanisms.

Origami's rich history and research in design optimization can provide an "orimimetic" perspective to LEM design that can lead to the generation of novel mechanisms.

\section{Objective}

Orimimetic design refers to the application of the concepts of folding to mechanism design. It can provide alternative ways to achieve a particular range of motion; compliant mechanisms have a similar aim. In addition, origami is achieved from the manipulation of a planar material which motivates its examination as a lamina emergent mechanism. The objective of this paper is to identify links between origami and compliant mechanisms to facilitate the application of origami design literature to compliant mechanism and LEM design.

\section{Nomenclature}

It is helpful to establish terminology to facilitate discussion. Key terms, and their use, are provided below.

\subsection{Origami}

Origami. Origami is defined as the art of folding paper, and in the context of engineering, it is the use of folding to solve mechanical problems (Demaine and O'Rourke, 2007).

Kirigami. Kirigami is defined as the art of folding and cutting paper (Hart, 2007). An example of kirigami is shown in Fig. 2 with the side view showing how creases are made such that the house pops out of the paper. Kinetic kirigami models are often LEMs, especially kirigami pop-up models.

Hinge creases. In traditional origami hinge creases define the boundaries between flaps. In the context of mechanisms they are creases along which lies an interface of two planar faces and it is the axis about which both facets rotate (Demaine and O'Rourke, 2007).

Construction creases. Creases used to create references in the construction of the mechanism are not directly used to create motion, are referred to as construction creases. In some cases, they coincide with hinge creases (Demaine and O'Rourke, 2007).

Structural creases. Structural creases are used to define the shape of flaps; they can be hinge creases as well. These creases are not needed in most mechanisms and they are not feasible for many materials. They may/can be substituted or eliminated in various ways (Demaine and O'Rourke, 2007).

Crease vs. fold. A fold is an action and a crease is the product of that action. Creases may be folded in one of two ways: mountain or valley.

Mountain crease. A convex crease (Hull, 2002).

Valley crease. A concave crease (Hull, 2002).

\subsection{Mechanisms}

Compliant mechanism. Mechanisms which transfer or transform motion, force, or energy at least in part through the deflection of flexible members are compliant mechanisms (Howell, 2001).

Lamina emergent mechanism. Lamina emergent mechanisms (LEMs) are a subset of compliant mechanisms made from planar materials (lamina) with motion that emerges out of the fabrication plane (Jacobsen et al., 2010). They are a subset of compliant mechanisms, in that they use the deflection of flexible members to achieve the desired motion. As a subset of compliant mechanisms, LEMs can provide feasible, repeatable solutions to advance the design and manufacturing of products. The advantages of LEMs include: reducing the number of parts, reducing cost, reducing weight, improving recyclability, increasing precision, and eliminating assembly (Jacobsen et al., 2010). The incorporation and use of LEMs offer many potential advantages in the design of mechanical products. These mechanisms can provide opportunities for more cost-effective, compact, easy to assemble, and modular products.

Spherical mechanism. In a spherical mechanism any point in a moving body is confined to move within a spherical surface, and all spherical surfaces of motion are concentric (Chiang, 1988). 

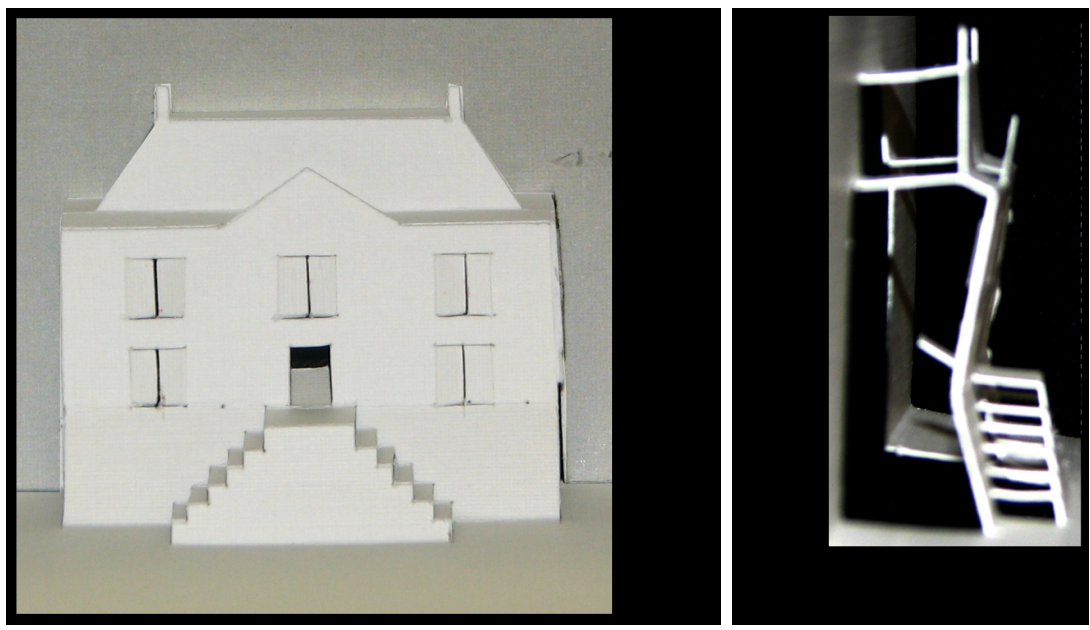

Figure 2. A kirigami house made in cardstock.

Orimimetic. Orimimetic means the ability to imitate folds. In an engineering design context, it refers to the ability to use the concept of folding to solve problems. Specifically it refers to the use of folding, either conceptually or literally, in mechanism design.

\subsection{Principles from graph theory}

Graph. A graph consists of points, called vertices, and connections, called edges (Marcus, 2008).

Planar graph. A graph with no edges crossing is a planar graph (Marcus, 2008).

Facet. The area enclosed by a planar graph is referred to as a facet.

Degree. The degree of a vertex is the number of edges that occur at that vertex (Marcus, 2008).

Degree sequence. The degree sequence of a graph is the degrees of each vertex listed in decreasing order (Marcus, 2008).

Regular graph, d-regular graph. A graph is considered regular if all its vertices are of the same degree. It can be referred to as a $d$-regular graph, where $d$ is a non-negative integer where each vertex has degree $d$ (Marcus, 2008).

Generically rigid. A graph is generically rigid if almost every realization of the graph is rigid. A realization of a graph is an assignment of coordinates to joints but without restrictions on edge lengths. Generic realizations avoid degeneracies such as having three points collinear, four points concyclic, or inducing parallel edges (Demaine and O'Rourke, 2007).

\section{Origami background}

These sections describe the relevant history and current applications of origami.

\subsection{Origami history}

The current origami movement began in the early 20th century, though historically origami has its beginning in several countries dating back to the Muromachi period, from 13331573 AD (Demaine and O'Rourke, 2007). Akira Yosihzawa and his origami works have been largely credited for the creative explosion in origami in the last century (Demaine and O'Rourke, 2007). Since the 1920's, origami design has become increasingly complex and varied. Initially origami was used to create figures and animals. As the art has progressed, the models have become increasingly complex, starting with a few to a few dozen steps to a few hundred or even a thousand steps. In the last twenty years new branches of origami have begun to be explored by more than artists; a growing number of mathematicians, educators, engineers and scientists have gathered to discuss the applications of origami in their respective fields (Demaine and O'Rourke, 2007).

Closely related to origami are other forms of paper engineering, such as kirigami, pop-up paper mechanisms, and origami architecture (Winder et al., 2009a).

\subsection{Origami applications in engineering and science}

Previous work has focused on studying the folding process to map the transition between initial and final states. An example is airbag folding designs which unfold smoothly from their flat starting state to their final volume (Cromvik, 2007). The Diffractive Optics Group at Lawrence Livermore Laboratory has worked on developing a telescope having a $100 \mathrm{~m}$ diameter lens that could collapse to fit into a space vehicle having a $4 \mathrm{~m}$ diameter and $10 \mathrm{~m}$ length. Lang used origami to identify a design that would fit and maintain the integrity of the surface when deployed into space (Heller, 2003; Wu and You, 2010; Wei and Dai, 2009). Origami has also been applied to the crash box of a car to improve energy absorbtion 


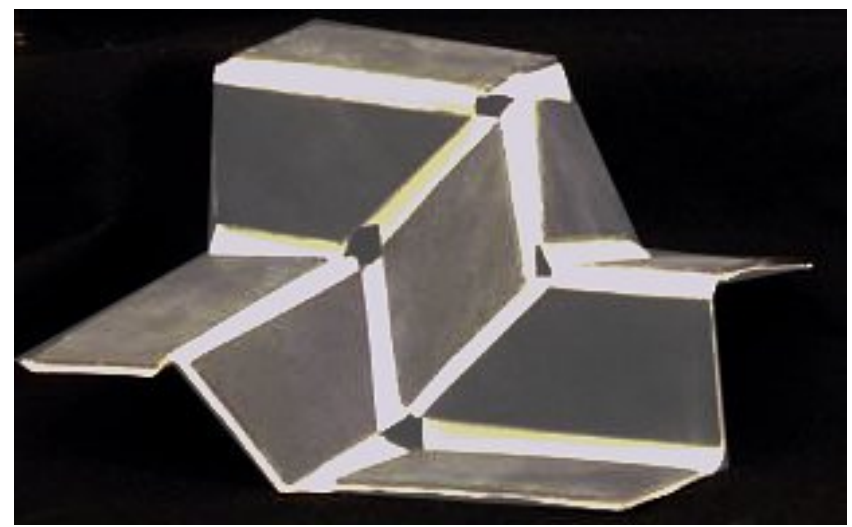

Figure 3. A common double slit kirigami representation and its corresponding PRBM both shown placed in the crease of a folded sheet of paper.

in a low speed collision (Ma and You, 2010). Miura explored methods of folding maps that could be unfolded with the simple pull of a corner (Miura, 2002). In the 1980's he invented the Miura-ori pattern, which is used as a basis for folding solar arrays (Miura and Natori, 1985). In 2005, Mahadevan published findings that this same pattern exists in leaf folding, wings, and flower petals (Mahadevan and Rica, 2005).

\section{Origami mechanisms}

This paper considers kinetic origami or origami mechanisms as opposed to origami structures. We will use the terms kinetic origami and origami mechanism interchangeably.

\subsection{Kinematic modeling of origami mechanisms}

Origami mechanisms are modeled with hinge creases as joints and facets as links (Winder et al., 2009b). Origami that is rigid-foldable is a "piecewise linear origami that is continuously transformable without the deformation of each facet" (Tachi, 2010). Rigid origami is also defined as having regions of the paper between crease lines that do not need to bend or twist in the folding process (i.e., the facet could be replaced with sheet metal and hinge creases replaced by hinges and it would still fold up) (Hull, 1994; Tachi, 2006). Figure 4 shows an example of rigid origami made by modeling the links of a square twist, a common origami unit for tessellations, converted to a polypropylene compliant mechanism.

\subsection{Origami and compliant mechanisms}

Origami mechanisms are compliant mechanisms; their motion is a result of the deflection of the material. As a material undergoes deformation, the resulting stored strain energy gives rise to an internal spring force. Balkcom notes that "the
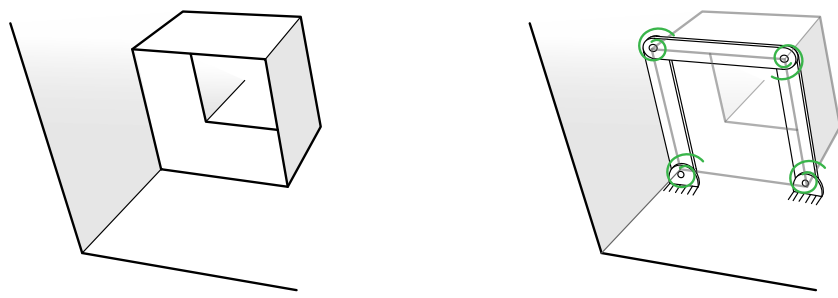

Figure 4. A rigid-foldable version of the square twist origami constructed from polypropylene sheet and paper. The polypropylene shows that it behaves rigidly, where bending is only allowed at the folds.

configuration of paper is determined by internal spring forces as well as external forces and constraints" (Balkcom and Mason, 2008). Thus, the origami can be viewed as a compliant mechanism which can be modeled using the pseudo-rigidbody model.

By focusing on origami in a fabricated state we only examine the hinge creases that contribute to the mechanism's structure and motion, not the folds that were used to construct it.

\section{Pseudo-rigid-body model}

The pseudo-rigid-body model (PRBM) uses rigid-body components, rigid links and springs, that have equivalent forcedeflection characteristics to model the deflection of flexible members (Howell, 2001). Figure 3 shows a common kirigami double slit and its corresponding PRBM (Winder et al., 2009a).

\subsection{Origami as a lamina emergent mechanism}

Because origami mechanisms are made from lamina materials it follows that they are LEMs. Traditionally, origami designs are judged by their efficiency and accuracy in terms of material usage and number of folds (Lang and Hull, 2005). For a model of an animal, efficiency is often defined by the ratio of the size of the final model to the initial sheet.

A branch of origami that is of special relevance to LEMs is flat-folding origami, which is origami that folds to a flat state, and tessellations or tilings, which could be useful for LEM applications in arrays. The repetition of basic folds in tessellations leads to the development of an origami mechanism that expands and contracts thereby capturing the motion that would be required for an array-type structure.

Table 1 lists examples of origami corollaries in each of the six categories. The potential for origami insights in LEM design can be seen by mapping existing applications of origami to the six application categories for LEMs (Albrechtsen et al., 2010). 
Table 1. Origami Applications by LEM Technology Category.

\begin{tabular}{ll}
\hline LEM Application Class & Origami Corollaries \\
\hline Disposable mechanisms & Packaging \\
\hline Novel Array mechanisms & $\begin{array}{l}\text { Space sails } \\
\text { Telescopes }\end{array}$ \\
\hline Scalable mechanisms & $\begin{array}{l}\text { Origami at nano-level } \\
\text { Cellular origami }\end{array}$ \\
\hline Surprising Motion mechanisms & Pop-up books \\
\hline Shock-absorbing mechanisms & Crash box \\
\hline Deployable mechanisms & $\begin{array}{l}\text { Airbags } \\
\text { Stents }\end{array}$ \\
\hline
\end{tabular}

\section{Linking origami to mechanisms with graphs}

Principles from graph theory can be used as a tool to understand how origami functions as a mechanism (Dobrjanskyj and Freudenstein, 1967). Graphs can model both origami and mechanisms on an abstract level and show their similarities. The abstraction of origami mechanisms to graphs demonstrates how the origami functions as a mechanism. Origami mechanisms can be reduced to graphs which show how they behave also. Applications of this are seen in Dai's carton folding research where graph theory is applied to show an equivalent mechanism (Dai and Jones, 2002).

In a graph of a kinetic origami model folds become line segments (edges) and links become nodes (vertices). From such graphs it can be seen that origami mechanisms may be thought of as interconnected linkages, with each loop representing a linkage system. It is important to note that in graph theory the shape of an edge or the position of the vertices does not matter since graphs depict connections (Marcus, 2008).

The crease pattern can be related to the graph of the origami mechanism in some cases where all creases are hinge creases and the origami can be flattened via actuation. In this case the graph and crease pattern are considered dual graphs. Figure 5 shows the square twist with its crease pattern and corresponding graph overlaid. However, for the general case, the crease pattern may include structural and construction creases which aid in the folding of the origami but do not contribute to the motion and therefore are not considered in the origami's corresponding graph.

Any simple planar connected graph with four segments connecting four vertices that is a 2-regular graph represents a four-bar mechanism. The graphs depict the degree of interconnection for each linkage system. This is shown in how each facet relates to its neighbouring facets. Each facet in a graph represents a linkage having the same number of links as nodes. For example, a facet (enclosed region) having four

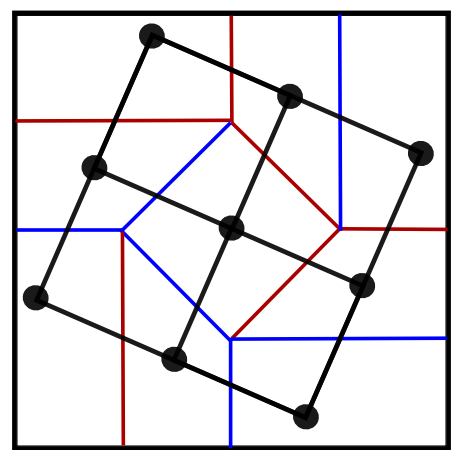

Figure 5. The crease pattern for the square twist shown in Fig. 4 is shown with its corresponding graph overlaid in black. In the crease pattern the red corresponds to mountain folds and blue to valley folds.

vertices (links) on the boundaries separating four segments (joints) is representative of a four-bar linkage.

\section{Paper mechanism examples}

Four paper mechanisms are shown in Table 2 with their corresponding kinematic and graphical representations. For simplification, all four paper mechanisms are flat-folding mechanisms and are rigid-foldable.

The kinematic representations for each mechanism examine a portion of the larger mechanism and its motion. When the paper mechanisms have a flat initial state and they can be represented with kinematics, by definition they can be realized as a LEM. In addition, the graphs representing each paper mechanism show more abstractly the mechanism and how they are coupled. The graphs also indicate the type of fold, where mountain folds are shown in red and valley folds are in blue.

The four-bar double slit mechanism (Table 2, row 1) uses the PRBM to show how each crease can be modeled as a joint with a torsional spring. Its graph is a 2-regular graph. It has three valley folds, and one mountain fold as indicated by the colors of edges in the graph. Each link has only two joints that define its motion.

For the 45-degree-fold twisting mechanism, also known as the pop-up spinner card (Table 2, row 2) each center fold, along the spine of the mechanism, is a 45 degree fold (O'Rourke, 2011). The kinematic representation shows only four links along the spine. The 45-degree-fold twisting mechanism is a chain of four-bar mechanisms where those links not on the ends share two links between each neighbouring linkage system, and each inside link is constrained by three joints with one of those joints shared between each neighbouring linkage system.

The square twist mechanism (Table 2, row 3) is a series of coupled spherical mechanisms which collapse onto one another. Figure 4 shows the basic square twist, and the 
Table 2. Table of Mechanisms.

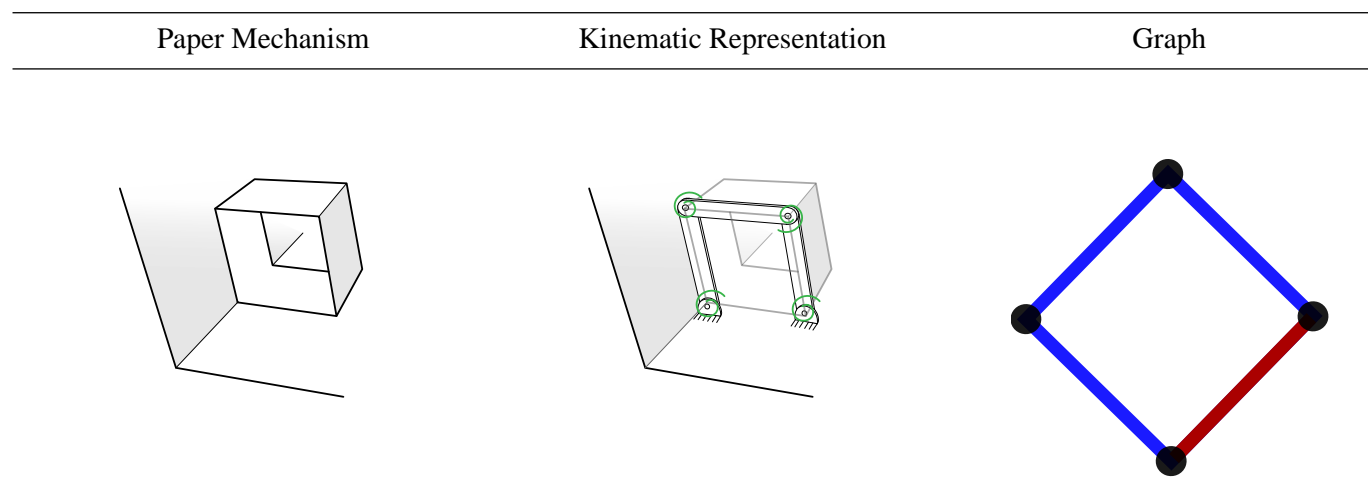

1. Four-Bar Double Slit
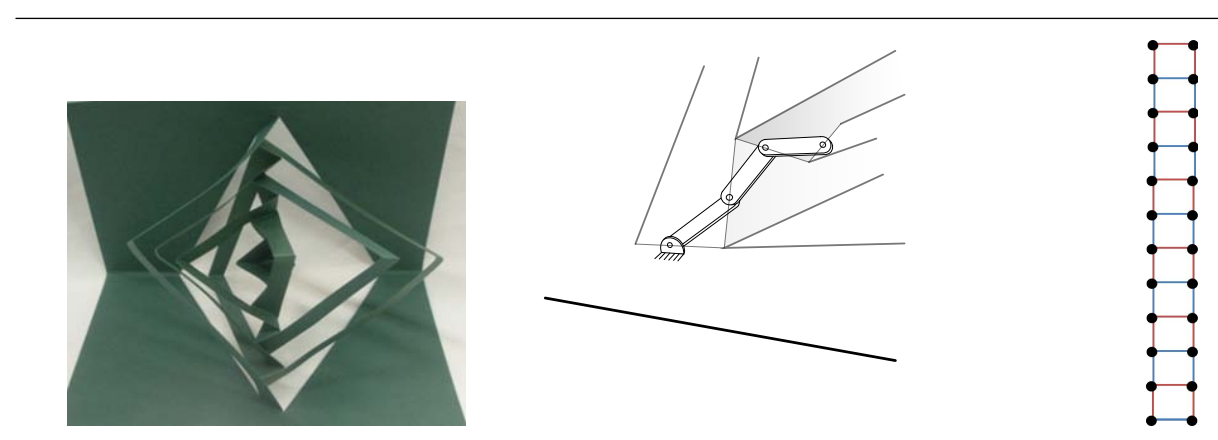

2. 45-degree-fold Twisting Mechanism
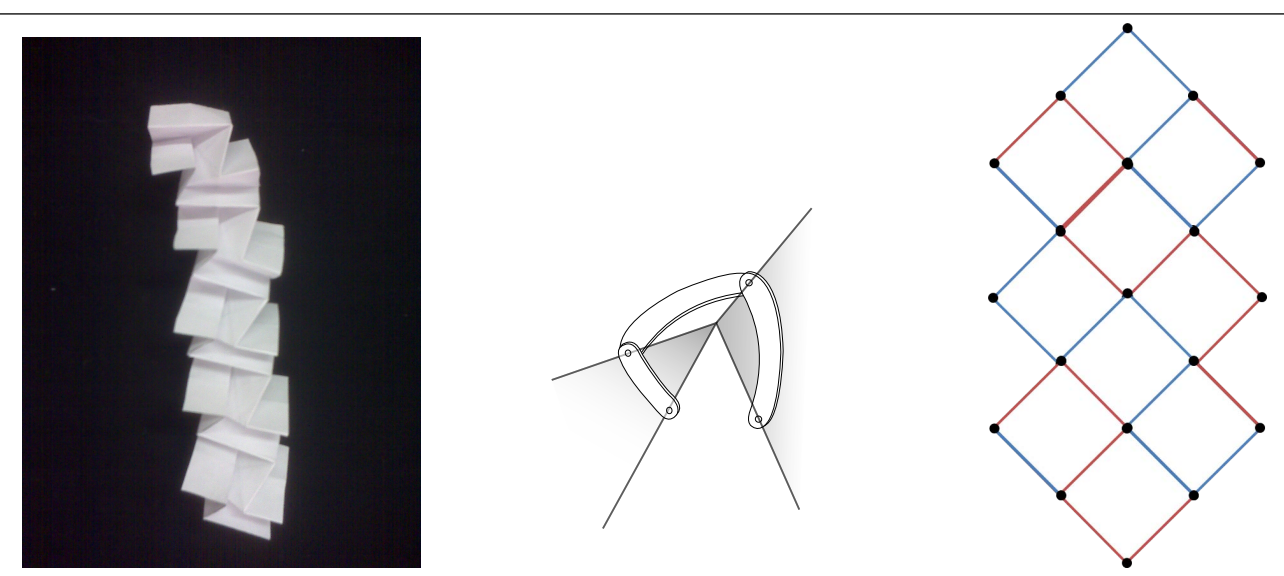

3. Square Twist
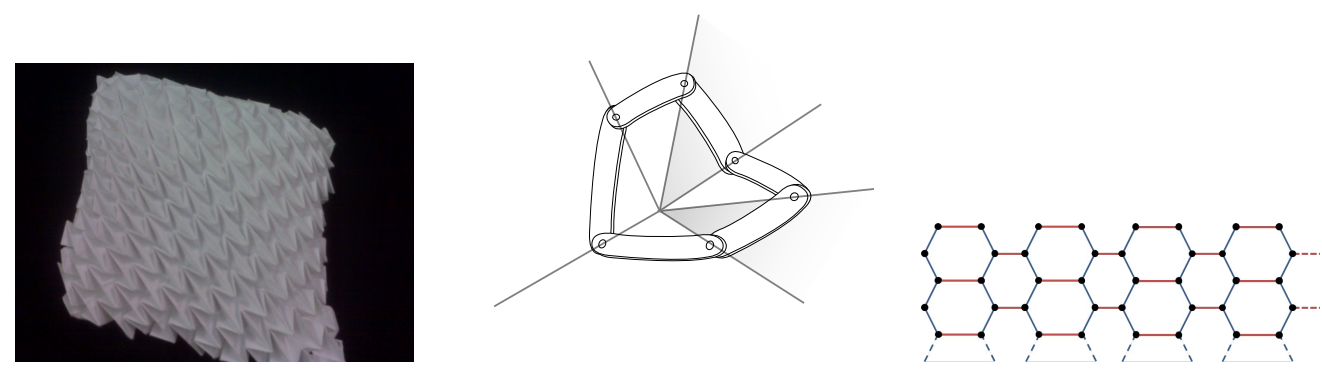

4. Water Bomb Base Tessellation 
kinematic representation shows one of the four-bar linkages seen at the corner of the twisting square platform. It may be better to express the square twist as inter-connected four-bar spherical mechanisms, which may be seen in the graph representation. The outer corner links each connect to only two joints. There are two neighbouring links on each end that are connected to three joints and are shared between two linkage systems. Of the 7 nodes of degree four, 3 are shared between four linkage systems and 4 are shared between three linkage systems.

Lastly, the kinematic representation for the water bomb base tessellation is shown in Table 2, row 4. As a graph the water bomb base tessellation is a 3-regular graph. The water bomb base tessellation contains six-bar linkage systems that are interconnected. Each link is shared between three linkage systems via three joints. This is also the basic tessellation used in the origami stent design which allows for the mechanism to compact radially as well as lengthwise (Kuribayashi et al., 2006).

\section{Discussion}

The examples in Table 2 examine a single four-bar mechanism, four-bars connected in series, four bars in series and parallel, and a six-bar spherical mechanism. The use of graph theory helps bridge the fields of origami and mechanisms. Visually the graphs show how the origami models are interconnected spherical mechanisms. The following sections will introduce concepts for knowing whether a graph can be realized as a flat-folding mechanism.

\subsection{Mobility}

Given any generic graph we can determine whether it is generically rigid or flexible and subsequently realizable as a mechanism or a structure, where a mechanism would be classified as generically flexible and a structure as generically rigid. A generic graph can be realized as a mechanism in two dimensions when

$e<2 n-3$

or in three dimensions when

$e<3 n-6$

where $n$ is the number of nodes and $e$ is the number of edges in the graph (Demaine and O'Rourke, 2007). When

$e=2 n-3$

the graph is generically rigid.

\subsection{Assembly}

For a four-bar mechanism to be assembled it is required to meet the criteria of

$l<s+p+q$ where $l$ refers to the length of the longest link, $s$ to the length of the shortest link and $p$ and $q$ the lengths of the other links. Likewise for an origami to be folded flat it must follow certain criteria. In determining flat-foldability it is assumed models can be folded perfectly, ignoring folding error, that paper has zero thickness, and that our creases have no width (Hull, 2002). The following criteria were developed for determining local flat-foldability, where local means about a single vertex.

Theorem 1 (Kawasaki, Justin, Hull). A single-vertex crease pattern defined by angles $\theta_{1}+\theta_{2}+\ldots+\theta_{n}=360^{\circ}$ is flatfoldable if and only if $n$ [the number of creases] is even and the sum of the odd angles $\theta_{2 i+1}$ is equal to the sum of the even angles $\theta_{2 i}$, or equivalently either sum is equal to $180^{\circ}$ (Demaine and O'Rourke, 2007).

This theorem is generally known as Kawasaki's Theorem, though also discovered independently by Justin in 1989. It's analogy in mechanisms is the condition for a changepoint mechanism where

$s+l=p+q$

Theorem 2 (Maekawa [Kasahara and Takahama], Justin). In a flat-foldable single-vertex mountain-valley pattern defined by angles $\theta_{1}+\theta_{2}+\ldots+\theta_{n}=360^{\circ}$, the number of mountains and the number of valleys differ by \pm 2 (Demaine and O'Rourke, 2007).

Theorem 2 is generally know as Maekawa's Theorem. It is essentially a condition for assembling a four-bar closed kinematic chain.

Theorem 3 (Kawasaki, Justin). If an angle $\theta_{i}$ is a strict local minimum (i.e., $\theta_{i-1}>\theta_{i}<\theta_{i+1}$ ), then the two creases bounding angle $\theta_{i}$ have an opposite mountain-valley assignment in any flat-foldable mountain-valley pattern (Demaine and O'Rourke, 2007).

This theorem is analogous to the conditions defining the classes for spherical four-bar LEMs where the shortest link has to connect to two links having opposite direction assignment.

An additional criterion is that a sheet can never penetrate a fold. To determine this it is required to have the geometry specified as well as the angles.

While criteria exist for determining the local flatfoldability (for a single vertex crease pattern), determining global flat-foldability is np-hard (non-deterministic polynomial-time hard). However we will explore the flatfoldability of the square twist to better understand these rules for assembly.

\section{Square twist}

The square twist crease pattern, shown in Fig. 6, has $2^{12}$ possible assignments and of those only 16 are flat-foldable (Hull, 2003). Figure 7 shows the 16 valid assignments for the square twist. It should be noted that only 6 of these 16 are unique. 


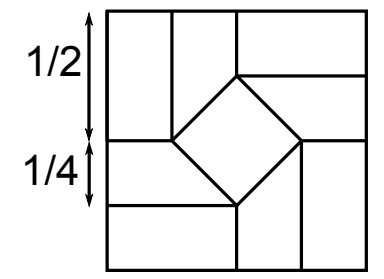

Figure 6. Crease pattern for square twist.
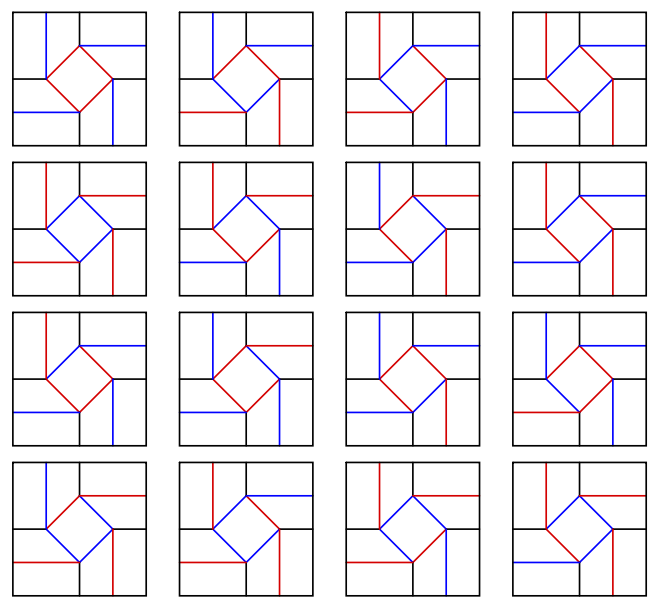

Figure 7. The 16 possible square twists given Theorem 3.

Next by applying Theorem 2 we can determine the remaining crease designations which are shown in Fig. 8.

It is easier to use the graph (on the right) as opposed to the crease pattern for visualizing how Theorem 2 is applied. Note that each subgraph for one of the four four-bars has either 3 mountain and 1 valley folds or 1 mountain and 3 valley folds. Corresponding paper representations of each are shown in Fig. 9.

All six paper models in Fig. 9 are flat-folded versions of the square twist which are kinetic origami. An important distinction to make is that while an origami can be folded flat it does not mean that it is kinetic. Since all folds here are simple folds and they are non-locking folds this allows us to actuate the square twist to have the twisting motion to go from a folded state (half the size of the original square) back to the original square.

\section{Conclusions}

Graphs of kinetic origami and kirigami models were used to show how the fields of origami and mechanisms are related. We propose that some of the literature from the origami field can be applied to mechanisms and vice versa. This was shown by comparing the criteria for flat-foldability to some conditions required for changepoint mechanisms to be as-
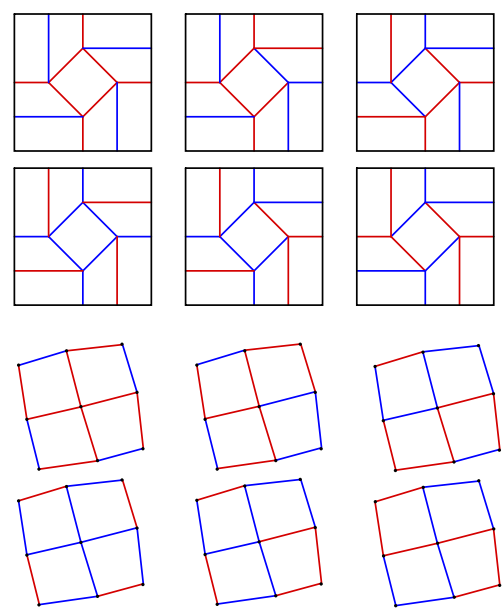

Figure 8. The 6 possible unique square twist crease patterns and their corresponding graphs given Theorem 2 .
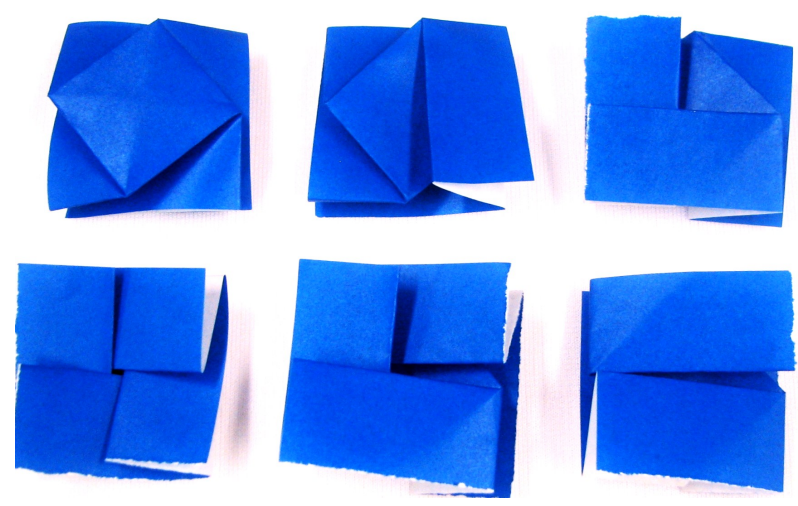

Figure 9. Folded origami models corresponding to the 6 possible square twists corresponding to those shown in Fig. 8.

sembled. As such it is anticipated that the abstraction to graph theory will improve the ability to synthesize LEMs.

In addition, by examining the graphs of some paper mechanisms it is understood that their motion is achieved because they are a system of coupled, or interconnected, mechanisms. Graph theory includes theories for identifying whether a generic graph is rigid or flexible in $n$-dimensions. As such it is believed that given any generic graph we can know whether it represents a structure or mechanism. Showing the relationship between origami and mechanisms will facilitate the application of origami design principles in the design of compliant mechanisms and LEMs.

Acknowledgements. The authors would like to acknowledge Larrin Wada for his drawings. This material is based upon work supported by the National Science Foundation under Grant No. CMMI-0800606. Any opinions, findings, and conclusions or recommendations expressed in this material are those of the authors and do not necessarily reflect the views of the National Science Foundation. 
Edited by: J. Herder

Reviewed by: H. Stachel and another anonymous referee

\section{References}

Albrechtsen, N., Magleby, S. P., and Howell, L. L.: Using Technology Push Product Development to Discover Enabled Opportunities and Potential Applications for Lamina Emergent Mechanisms, in: Proceedings of the ASME International Design Engineering Technical Conferences, DETC2010-28531, ASME, 2010.

Balkcom, D. J. and Mason, M. T.: Robotic origami folding, Int. J. Robot. Res., 27, 613-627, doi:10.1177/0278364908090235, compendex, 2008.

Buchner, T.: Kinematics of 3D Folding Structures for Nanostructured Origami TM, Ph.D. thesis, Massachusetts Institute of Technology, 2003.

Chiang, C. H.: Kinematics of Spherical Mechanisms, Cambridge University Press, 1988.

Cromvik, C.: Numerical Folding of Airbags Based on Optimization and Origami, Master's thesis, Chalmers University of Technology and Göteborg University, Sweden, 2007

Dai, J. S. and Jones, J. R.: Kinematics and mobility analysis of carton folds in packing manipulation based on the mechanism equivalent, Proceedings of the Institution of Mechanical Engineers - Part C - J. Mech. Eng. Sci., 216, 959-970, doi:10.1243/095440602760400931, 2002.

Demaine, D. E., Dinh, G., Crimp, V. F. L., Golan, M., Hull, D. T., and Gould, V.: Independent Lens: Between the Folds, 2010.

Demaine, E. and O'Rourke, J.: Geometric Folding Algorithms, Cambridge University Press, 2007.

Dobrjanskyj, L. and Freudenstein, F.: Some applications of graph theory to the structural analysis of mechanisms, J. Eng. Ind., 89, 153-158, 1967.

Hart, G.: Modular Kirigami, San Sebastian Spain, 1-8, 2007.

Heller, A.: A Giant Leap for Space Telescopes, Science and Technology Review by Lawrence Livermore National Laboratory, 12-18, 2003.

Howell, L. L.: Compliant Mechanisms, Wiley-Interscience, 2001.

Hull, T.: On the mathematics of flat origamis, Congressus numerantium, p. 215, 1994.

Hull, T.: The Combinatorics of Flat Folds: A Survey, in: Origami3: Third International Meeting of Origami Science, Mathematics, and Education, A K Peters, Ltd., 29-38, 2002.

Hull, T.: Counting Mountain-Valley Assignments for Flat Folds, Ars Combinatoria, 67, 175-178, 2003.

Jacobsen, J. O., Winder, B. G., Howell, L. L., and Magleby, S. P.: Lamina emergent mechanisms and their basic elements, Journal of Mechanisms and Robotics, 2, 1-9, doi:10.1115/1.4000523, compendex, 2010.

Kuribayashi, K., Tsuchiya, K., You, Z., Tomus, D., Umemoto, M., Ito, T., and Sasaki, M.: Self-Deployable Origami Stent Grafts as a Biomedical Application of Ni-rich TiNi Shape Memory Alloy Foil, Mat. Sci. Eng. A-Struct., 419, 131-137, 2006.
Lang, R. J. and Hull, T. C.: Origami design secrets: mathematical methods for an ancient art, The Mathematical Intelligencer, 27, 92-95, doi:10.1007/BF02985811, 2005.

Ma, J. and You, Z.: The Origami Crash Box, in: Origami 5: the 5th International Conference on Origami in Science Mathematics and Education, 2010.

Mahadevan, L. and Rica, S.: Self-Organized Origami, Science, 307, 1740, doi:10.1126/science.1105169, 2005.

Marcus, D. A.: Graph theory: a problem oriented approach, MAA, 2008.

Miura, K.: The Application of Origami Science to Map and Atlas Design, in: Origami3: Third International Meeting of Origami Science, Mathematics, and Education, edited by: Hull, T., A K Peters, Ltd., 2002.

Miura, K. and Natori, M.: 2-D Array Experiment on Board a Space Flyer Unit, Space Power, 5, 345-356, 1985.

O'Rourke, J.: How To Fold It, Cambridge University Press, 2011.

Tachi, T.: Simulation of Rigid Origami, in: Origami 4: Fourth International Meeting of Origami Science, Mathematics, and Education, edited by: Lang, R., A K Peters, Ltd., 175-187, 2006.

Tachi, T.: Rigid-Foldable Thick Origami, in: Origami 5: the 5th International Conference on Origami in Science Mathematics and Education, 2010.

Watanabe, N. and Kawaguchi, K.-I.: The Method for Judging Rigid Foldability, in: Origami 4: Fourth International Meeting of Origami Science, Mathematics, and Education, edited by: Lang, R., 165-174, 2006.

Wei, G. and Dai, J.: Geometry and kinematic analysis of an origami-evolved mechanism based on artmimetics, in: Reconfigurable Mechanisms and Robots, 2009, ReMAR 2009. ASME/IFToMM International Conference on, 450-455, 2009.

Winder, B. G., Magleby, S. P., and Howell, L. L.: A study of joints suitable for lamina emergent mechanisms, in: 2008 ASME International Design Engineering Technical Conferences and Computers and Information in Engineering Conference, DETC 2008, 3-6 August 2008, vol. 2 of 2008 Proceedings of the ASME International Design Engineering Technical Conferences and Computers and Information in Engineering Conference, DETC 2008, ASME, New York City, NY, United states, compendex, 339-349, 2009a.

Winder, B. G., Magleby, S. P., and Howell, L. L.: Kinematic representations of pop-up paper mechanisms, Journal of Mechanisms and Robotics, 1, 1-10, doi:10.1115/1.3046128, compendex, 2009b.

$\mathrm{Wu}, \mathrm{W}$. and You, Z.: Modelling rigid origami with quaternions and dual quaternions, Proceedings of the Royal Society A: Mathematical, Physical and Engineering Science, 466, 2155-2174, doi:10.1098/rspa.2009.0625, 2010. 